\title{
Bone marrow stromal cell sheets may promote axonal regeneration and functional recovery with suppression of glial scar formation after spinal cord transection injury in rats
}

\author{
Akinori Okuda, MD, ${ }^{1,2}$ Noriko Horii-Hayashi, PhD, ${ }^{2}$ Takayo Sasagawa, MS, ${ }^{2}$ \\ Takamasa Shimizu, MD, PhD, ${ }^{1}$ Hideki Shigematsu, MD, PhD, ${ }^{1}$ Eiichiro Iwata, MD, PhD, ${ }^{1}$ \\ Yasuhiko Morimoto, MD, ${ }^{1}$ Keisuke Masuda, MD, ${ }^{1}$ Munehisa Koizumi, MD, PhD, ${ }^{3}$ \\ Manabu Akahane, MD, PhD, ${ }^{4}$ Mayumi Nishi, MD, PhD, ${ }^{2}$ and Yasuhito Tanaka, MD, PhD ${ }^{1}$ \\ 1Department of Orthopaedic Surgery, Nara Medical University, Kashihara; ${ }^{2}$ Department of Anatomy and Cell Biology, Faculty of \\ Medicine, Nara Medical University, Kashihara; ${ }^{3}$ Spine and Spinal Cord Surgery Center, Nara Prefecture General Medical Center; \\ and ${ }^{4}$ Department of Public Health, Health Management, and Policy, Nara Medical University, Kashihara, Nara, Japan
}

\begin{abstract}
OBJECTIVE Transplantation of bone marrow stromal cells (BMSCs) is a theoretical potential as a therapeutic strategy in the treatment of spinal cord injury (SCl). Although a scaffold is sometimes used for retaining transplanted cells in damaged tissue, it is also known to induce redundant immunoreactions during the degradation processes. In this study, the authors prepared cell sheets made of BMSCs, which are transplantable without a scaffold, and investigated their effects on axonal regeneration, glial scar formation, and functional recovery in a completely transected SCl model in rats.
\end{abstract}

METHODS BMSC sheets were prepared from the bone marrow of female Fischer 344 rats using ascorbic acid and were cryopreserved until the day of transplantation. A gelatin sponge (GS), as a control, or BMSC sheet was transplanted into a 2-mm-sized defect of the spinal cord at the T-8 level. Axonal regeneration and glial scar formation were assessed 2 and 8 weeks after transplantation by immunohistochemical analyses using anti-Tuj1 and glial fibrillary acidic protein (GFAP) antibodies, respectively. Locomotor function was evaluated using the Basso, Beattie, and Bresnahan scale.

RESULTS The BMSC sheets promoted axonal regeneration at 2 weeks after transplantation, but there was no significant difference in the number of Tuj1-positive axons between the sheet- and GS-transplanted groups. At 8 weeks after transplantation, Tuj1-positive axons elongated across the sheet, and their numbers were significantly greater in the sheet group than in the GS group. The areas of GFAP-positive glial scars in the sheet group were significantly reduced compared with those of the GS group at both time points. Finally, hindlimb locomotor function was ameliorated in the sheet group at 4 and 8 weeks after transplantation.

CONCLUSIONS The results of the present study indicate that an ascorbic acid-induced BMSC sheet is effective in the treatment of $\mathrm{SCl}$ and enables autologous transplantation without requiring a scaffold.

https://thejns.org/doi/abs/10.3171/2016.8.SPINE16250

KEY WORDS bone marrow stromal cell; cell sheet; axonal regeneration; spinal cord injury; ascorbic acid; glial scar

ABBREVIATIONS AscP = L-ascorbic acid phosphate; $\mathrm{BBB}=$ Basso, Beattie, and Bresnahan; BMSC = bone marrow stromal cell; DAPI = 4',6-diamidino-2-phenylindole; $\mathrm{ECM}$ = extracellular matrix; GFAP = glial fibrillary acidic protein; GS = gelatin sponge; PBS = phosphate-buffered saline; PBST = PBS with Triton X-100; SCI = spinal cord injury.

SUBMITTED March 1, 2016. ACCEPTED August 31, 2016.

INCLUDE WHEN CITING Published online November 25, 2016; DOI: 10.3171/2016.8.SPINE16250. 
A CCUMULATING evidence suggests that transplantation of adult bone marrow stromal cells (BMSCs) is a theoretical potential as a therapeutic strategy in the treatment of spinal cord injury (SCI). BMSCs are easily isolated and harvested in vitro and can be cryopreserved for a long duration..$^{21}$ BMSCs prepared from patients enable autologous transplantation. Previous studies have shown that BMSCs enhanced axonal regeneration and suppressed glial scar formation in both contused $\mathrm{d}^{10,16,19 \text {, }}$ ${ }^{28,30}$ and transected ${ }^{33} \mathrm{SCI}$ models. In the case of the transected model, a scaffold was still needed to fill the defect of the spinal cord as well as to retain the transplanted cells at the site. ${ }^{33}$ Many kinds of scaffolds have been developed to date, including self-assembled peptide nanofibers, ${ }^{15}$ gelatin, ${ }^{7}$ collagen ${ }^{26}$ Matrigel, ${ }^{18}$ and fibrin..${ }^{17}$ However, a previous report demonstrated that these biodegradable scaffolds induce inflammatory reactions during their degradation. ${ }^{32}$ Thus, an autologous cell sheet that is transplantable without scaffolds is considered to be a useful material for the treatment of SCI.

BMSCs produce abundant extracellular matrix (ECM) proteins, which are considered suitable for tissue engineering applications. ${ }^{25}$ Our previous study demonstrated that the addition of L-ascorbic acid phosphate (AscP) to the culture medium for BMSCs allowed the cells to disperse into a sheet form. ${ }^{20}$ In the same study, the BMSC sheets were found to produce various growth factors, such as vascular endothelial growth factor, basic fibroblast growth factor, platelet-derived growth factor, and insulin-like growth factor $-1,{ }^{20}$ which have been reported to enhance axonal regeneration and to confer neuroprotection..$^{11,12,31}$ Thus, we hypothesized that transplanting a BMSC sheet into a spinal cord defect without a scaffold might enhance axonal regeneration and suppress glial scar formation. The aim of this study was to assess the usefulness of BMSC sheets in terms of axonal regeneration and the suppression of glial scar formation in a completely transected SCI model. We first examined the cell viability of BMSC sheets after recovery from cryopreservation and investigated their ECM components by immunocytochemical staining. Second, we examined whether the sheets are permissive for axonal elongation and the extension of astrocyte processes, using neurospheres cocultured on the sheet, because previous work has shown that the permissiveness of transplants for astrocyte processes is also important to prevent glial scar formation in vivo. ${ }^{29}$ Third, we investigated the number of regenerating axons and glial scar formation by immunohistochemical staining after transplantation of the BMSC sheet into a spinal cord defect. Finally, functional recovery was assessed using the Basso, Beattie, and Bresnahan (BBB) locomotor rating scale. ${ }^{5}$

\section{Methods}

\section{Animals}

All experimental procedures were approved by the Institutional Animal Care and Use Committee of Nara Medical University. Female Fischer 344 rats were used in all experiments and were purchased from Japan SLC Inc. A total of 30 rats were used for the experiments; 18 were used for histological and motor functional analyses (8-week-old rats), 10 were used for collecting bone marrow (7-week-old rats), and 2 embryos (embryonic Day 17) were used for preparing neurospheres.

\section{BMSC Sheets}

The preparation of BMSCs from the femoral bone was performed as described previously, ${ }^{2,3,23}$ and their application to cell sheets followed the methods described in our previous study. ${ }^{20}$ In brief, bone marrow cells were obtained from the femoral shafts with standard culture medium: minimal essential medium (Nacalai Tesque) containing $15 \%$ fetal bovine serum (JRH Bioscience Inc.) and antibiotics $(100 \mathrm{U} / \mathrm{ml}$ of penicillin and $100 \mu \mathrm{g} / \mathrm{ml}$ of streptomycin, Nacalai Tesque). The released cells were collected in T-75 flasks (BD Falcon, BD Biosciences) with the medium and maintained in a humidified atmosphere with $5 \% \mathrm{CO}_{2}$ at $37^{\circ} \mathrm{C}$. After reaching confluence, the cells were trypsinized and seeded in 6 - $\mathrm{cm}$ dishes $(60 \times 20 \mathrm{~mm}$; BD Falcon $)$ at $1 \times 10^{4}$ cells $/ \mathrm{cm}^{2}$ with the same medium containing $0.28 \mathrm{mM}$ of AscP magnesium salt n-hydrate (Wako Pure Chemical Industries). After 14 days in culture, the cells reached confluence and formed a sheet. The cell sheets were cryopreserved with CryoScarless medium (DMSOFree, BioVerde, Inc.) according to the manufacturer's instructions, and maintained at $-80^{\circ} \mathrm{C}$ until transplantation.

\section{Neurite Growth and Glial Extension on BMSC Sheets}

Neurospheres were prepared from the cortices of embryonic Day 17 embryos as described previously. ${ }^{27}$ In brief, the tissues were dissociated by trituration with a firepolished Pasteur pipette, and the cells were suspended in neurosphere culture medium: Dulbecco's modified Eagle medium/Ham's F-12 (dilution 1:1, Gibco) containing 1\% N2 supplement (Gibco), $1 \%$ penicillin/streptomycin (Nacalai Tesque), $0.2 \%$ recombinant human basic fibroblast growth factor (PeproTech), and 2\% B27 supplement (Thermo Fisher Scientific). Cells were seeded at $2 \times 10^{5}$ cells $/ \mathrm{ml}$ in T-75 flasks containing the medium and incubated in 5\% $\mathrm{CO}_{2}$ at $37^{\circ} \mathrm{C}$. After 1 week, neurospheres were collected, trypsinized, and passaged to secondary culture. After 3.5 days, the neurospheres were collected and cryopreserved with STEM CELL-BANKER (GMP-grade, Takara Bio Inc.) in liquid nitrogen until use. For coculture of neurospheres and a BMSC sheet, thawed neurospheres were plated on BMSC sheets attached to poly-D-lysine-coated coverslips in a 24 -well plate and maintained in neurosphere culture medium for 3.5 days in $5 \% \mathrm{CO}_{2}$ at $37^{\circ} \mathrm{C}$.

\section{Cell Viability Assay}

Cryopreserved BMSC sheets were thawed at $37^{\circ} \mathrm{C}$ and rinsed twice with the standard culture medium. Trypsinized cells were centrifuged at $100 \mathrm{~g}$ for 5 minutes at $4^{\circ} \mathrm{C}$. The cell pellets were resuspended with the medium and stained with trypan blue solution (Nacalai Tesque). Cell counting was performed under a microscope using a hemocytometer. Cell viability was defined as follows: [(total number of cells counted - number of stained cells)/total number of cells counted] $\times 100$. The viability of thawed BMSC sheets was compared with that of fresh BMSC sheets. 


\section{SCI Surgery}

Cryopreserved BMSC sheets were thawed on the day of surgery as described above and were maintained in the standard culture medium until transplantation. Animals were anesthetized with $2.0 \%$ isoflurane in $2.0 \mathrm{~L} / \mathrm{min}$ oxygen and subjected to laminectomy at the T-8 level, leaving the dura mater intact. The spinal cord was completely transected at 2 points $2 \mathrm{~mm}$ apart with microsurgical scissors, and the fragment of the spinal cord was removed. A BMSC sheet or gelatin sponge (GS) was transplanted into the defect to fill the space between the rostral and caudal stumps of the spinal cord. After transplantation, the muscles and skin were sutured layer by layer. Food and water were provided ad libitum. The bladder was pressed twice a day until spontaneous voiding began.

\section{Assessment of Locomotor Function}

All locomotor assessments were performed by at least 2 examiners blinded to the group allocations. The hindlimb locomotor functions of animals in both the BMSC sheet group (sheet group) and GS group were assessed using the BBB locomotor scale ${ }^{5}$ at $1,2,4$, and 8 weeks after transplantation. The BBB scale (from 0 to 21 points) is used to assess locomotor recovery, including joint movements, stepping ability, coordination, and trunk stability. A score of 21 indicates unimpaired locomotion, as observed in uninjured rats.

\section{Immunostaining}

Histological and cytological immunostainings were performed as described previously. ${ }^{13,14}$ In brief, cultured cells plated on poly-D-lysine-coated coverslips were fixed with $4 \%$ paraformaldehyde in $0.1 \mathrm{M}$ phosphate-buffered saline (PBS) (pH 7.4) for 10 minutes. After permeabilization with $0.3 \%$ Triton X-100 in PBS (PBST), cells were blocked with 5\% normal horse serum in PBST and incubated with Alexa Fluor-546-conjugated phalloidin and the following primary antibodies: mouse anti-collagen I (1:100, Abcam), rabbit anti-collagen IV (1:100, Progen), guinea pig anti-laminin (1:200, gift from Dr. Miyata), ${ }^{22}$ mouse anti-Tuj1 (1:200, Covance), or rabbit anti-glial fibrillary acidic protein (GFAP; 1:1000, Abcam). They were further incubated with species-specific secondary antibodies conjugated with Alexa Fluor-488 (1:1000, Thermo Fisher) or CF-555 (1:1000, Biotium). The coverslips were shielded using Vectashield containing 4',6-diamidino2-phenylindole (DAPI, Vector).

For immunohistochemical analysis, animals were anesthetized with sodium pentobarbital $(100 \mathrm{mg} / \mathrm{kg})$ and transcardially perfused with PBS followed by $4 \%$ paraformaldehyde. Dissected T6-10 spinal cord segments were postfixed with the same fixative overnight. After cryoprotection with $30 \%$ sucrose in PBS, 20 - $\mu$ m-thick sagittal sections were made using a cryostat (Leica) and mounted on glass slides. The sections were treated with $25 \mathrm{mM}$ glycine in PBS, $0.3 \%$ PBST, and then blocked in PBST containing 5\% normal horse serum for 2 hours. The sections were incubated with the following primary antibodies for 2 days at $4^{\circ} \mathrm{C}$ : mouse anti-Tuj1 (1:200, Covance), rabbit anti-Tuj1 (1:200, Covance), mouse anti-GAP43 (1:100,

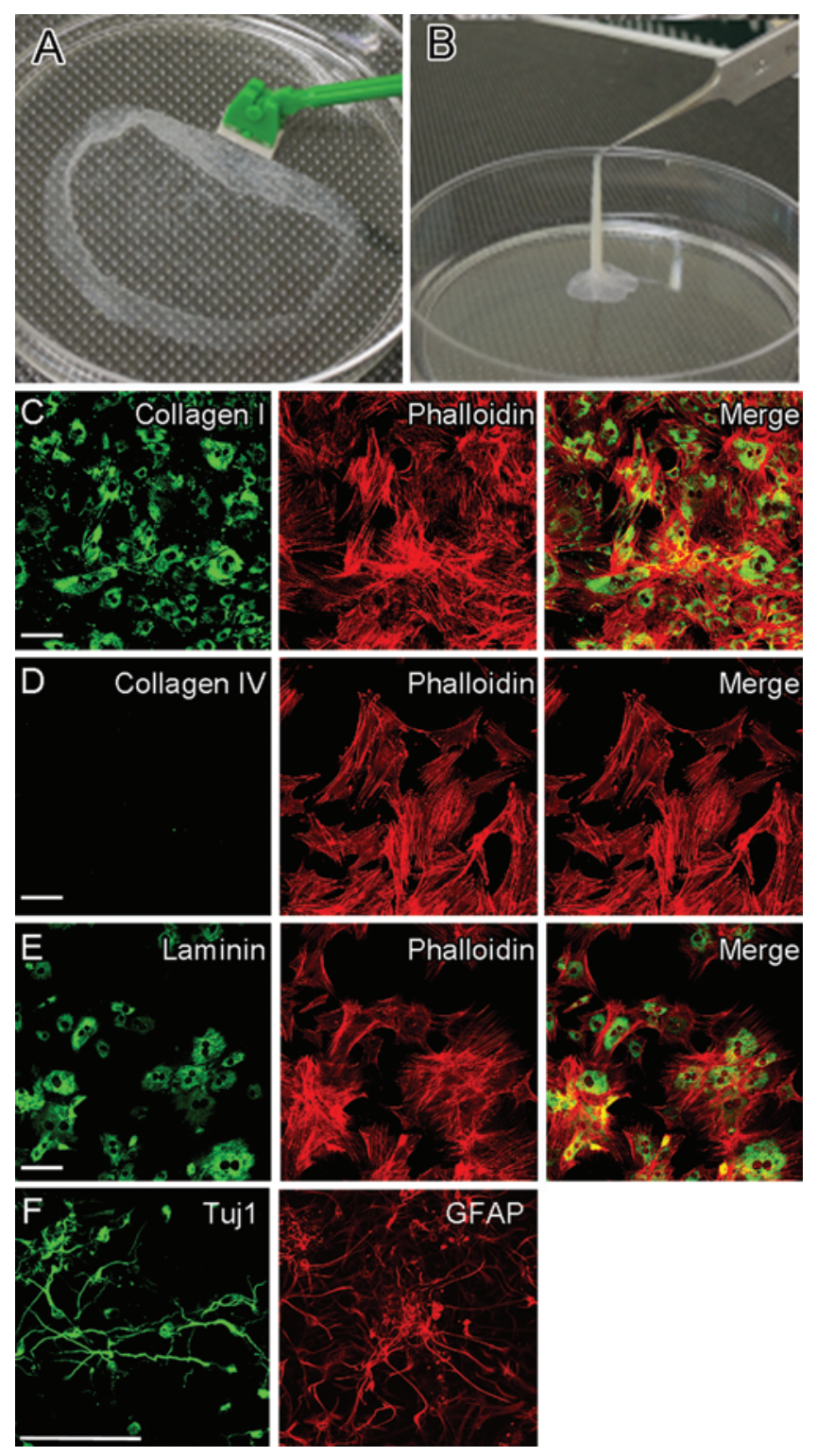

FIG. 1. BMSC sheets are permissive for axonal growth and astrocyte extension. A and B: The appearance of a BMSC sheet. The sheet could be scraped and picked up with forceps. C-E: Double labeling of ECM proteins, collagen Type I (C), collagen Type IV (D), laminin $(E)$, and actin with phalloidin in BMSC sheets. The sheets were immunoreactive for collagen Type I and laminin, but not for collagen Type IV. F: Neurospheres were cultured on a BMSC sheet for 3.5 days. Immunocytochemistry using Tuj1 and GFAP revealed extending Tuj1positive neurites and processes of GFAP-positive astrocytes. Bar $=100$ $\mu \mathrm{m}$. Figure is available in color online only.

Millipore), rabbit anti-GFAP (1:1000, Abcam), or guinea pig anti-laminin. The sections were incubated with species-specific secondary antibodies conjugated with Alexa Fluor-488 or CF-555 (1:1000, Biotium) for 2 hours. The coverslips were shielded using Vectashield containing DAPI. All immunocytochemical and immunohistochemical images were captured using a FluoView 1000 confocal microscope (Olympus) with a $512 \times 512$-pixel array. 


\section{Quantification of Axonal Regeneration and Glial Scarring}

Axonal regeneration and glial scar formation were analyzed using the morphometric software program Metamorph (Molecular Devices). Nine serial sections prepared at $80-\mu \mathrm{m}$ intervals including the midline were analyzed per animal. The numbers of regenerating axons were quantified by counting the numbers of Tuj1-positive axons with a length of at least $50 \mu \mathrm{m}$ within the 2 rectangles $(500 \times$ $1000 \mu \mathrm{m})$ that were $400 \mu \mathrm{m}$ away from the rostral and caudal stumps of the injury sites. Glial scar formation was quantified by measuring the GFAP-positive areas within the 2 rectangles $(1250 \times 500 \mu \mathrm{m})$ including the rostral and caudal edges of the injury sites. The numbers of axons and GFAP-positive areas obtained from 9 sections were summed as the final numbers and areas, respectively.

\section{Statistical Analysis}

The Kolmogorov-Smirnov test, Shapiro-Wilk test, Ftest, t-test, or Welch's test was performed depending on whether the data followed a normal distribution. The t-test was performed to examine significant differences in glial scar area, and Welch's test was performed to determine significant improvements in axonal regeneration. Furthermore, the t-test and repeated-measures analysis of variance were performed to examine significant improvements in BBB scores. All data are presented as the mean \pm SEM. Data analyses were conducted using the SPSS software package (version 23.0, IBM); $p<0.05$ was considered to denote statistical significance.

\section{Results}

\section{Cell Viability of Cryopreserved BMSC Sheets}

The cell viability of the BMSC sheets after cryopreservation was $92.88 \% \pm 1.32 \%(\mathrm{n}=7)$, which was considered to represent good recovery after freezing. We therefore used cryopreserved BMSC sheets for all transplantation experiments.

\section{BMSC Sheets Provided Neural Cells With a Permissive Environment for Elongation In Vitro}

BMSC sheets prepared using AscP were easily detached from the culture dishes with a cell scraper (Fig. 1A) and could be lifted up with forceps (Fig. 1B). Immuno- histochemical results showed that BMSC sheets expressed collagen Type I and laminin (Fig. 1C and 1E), but not collagen Type IV (Fig. 1D). Immunostaining of neurospheres cultured on a BMSC sheet revealed that both Tuj1-positive neurites and GFAP-positive astrocyte processes could elongate on the sheet (Fig. 1F). These results indicated that the BMSC sheets do not inhibit the elongation of axons and astrocyte processes.

\section{BMSC Sheets Promoted Axonal Regeneration}

A spinal cord defect (Fig. 2A) was filled with a folded BMSC sheet (Fig. 2B) or GS (Fig. 2C). Two weeks after transplantation, double labeling of Tuj1 and GAP43 (a marker for growing axons) was performed. The results showed that the majority of Tuj1-positive axons at the sheet-transplanted site overlapped with GAP43-positive axons (Fig. 3A), indicating that almost all axons at the injured site were regenerating axons.

Double labeling of Tuj1 and GFAP was performed at 2 and 8 weeks after transplantation. More Tuj1-positive axons beyond the GFAP-positive glial scar were observed in sheet-transplanted rats compared with GS-transplanted rats at 2 weeks after transplantation (Fig. 3B). At 8 weeks after transplantation, elongated Tuj1-positive axons crossed the transplanted site in the sheet-transplanted animals, but not in the GS-transplanted animals (Fig. 3C). The number of Tuj1-positive axons (green boxes in Fig. $3 \mathrm{D})$ was significantly greater in the sheet group than in the GS group at 8 weeks after transplantation (Fig. 3E; BMSC, $429 \pm 97.17$; GS, $118 \pm 19.84 ; \mathrm{p}=0.047, \mathrm{n}=4$ per group). However, there was no significant difference ( $\mathrm{p}=$ 0.124 ) between the sheet and GS groups at 2 weeks after transplantation (Fig. 3E; BMSC, $64.75 \pm 25.00$; GS, 12.25 $\pm 5.79, \mathrm{n}=4$ per group). These results indicate that BMSC sheets promote axonal regeneration after SCI.

\section{BMSC Sheets Attenuated Glial Scar Formation and Affected the Shape of the Glial Scar}

GFAP immunoreactivity in the rostral and caudal spinal cord stumps appeared weaker in the sheet-transplanted rats compared with those of the GS-transplanted rats at 2 and 8 weeks after transplantation (Fig. 4A and 4B). Furthermore, GFAP immunoreactivity at 8 weeks after transplantation showed an irregular and straggly

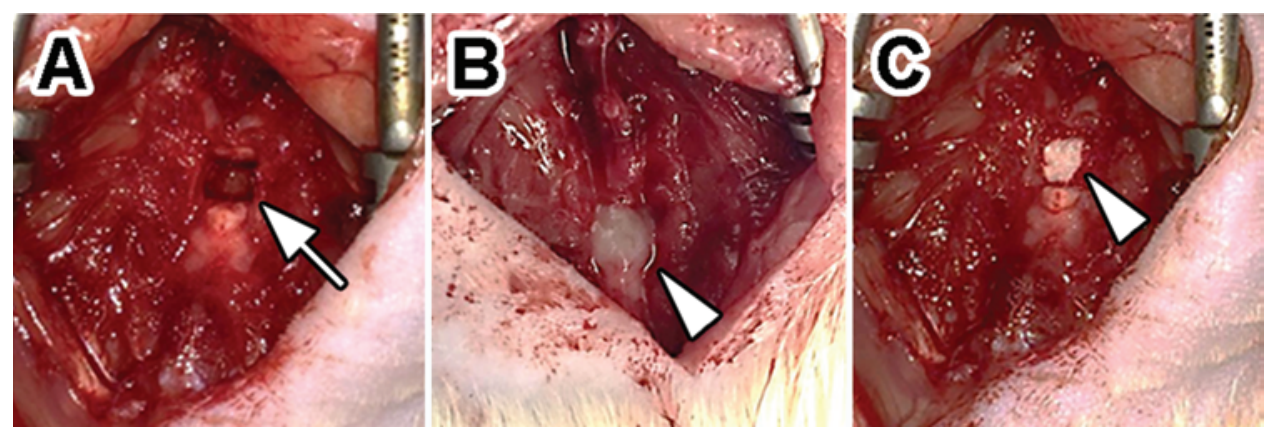

FIG. 2. A T-8 laminectomy was performed, and the spinal cord was transected at the same level with a 2-mm defect (A, arrow). A BMSC sheet (B) or GS (C) was transplanted into the defect in contact with both spinal stumps (arrowheads). Figure is available in color online only. 

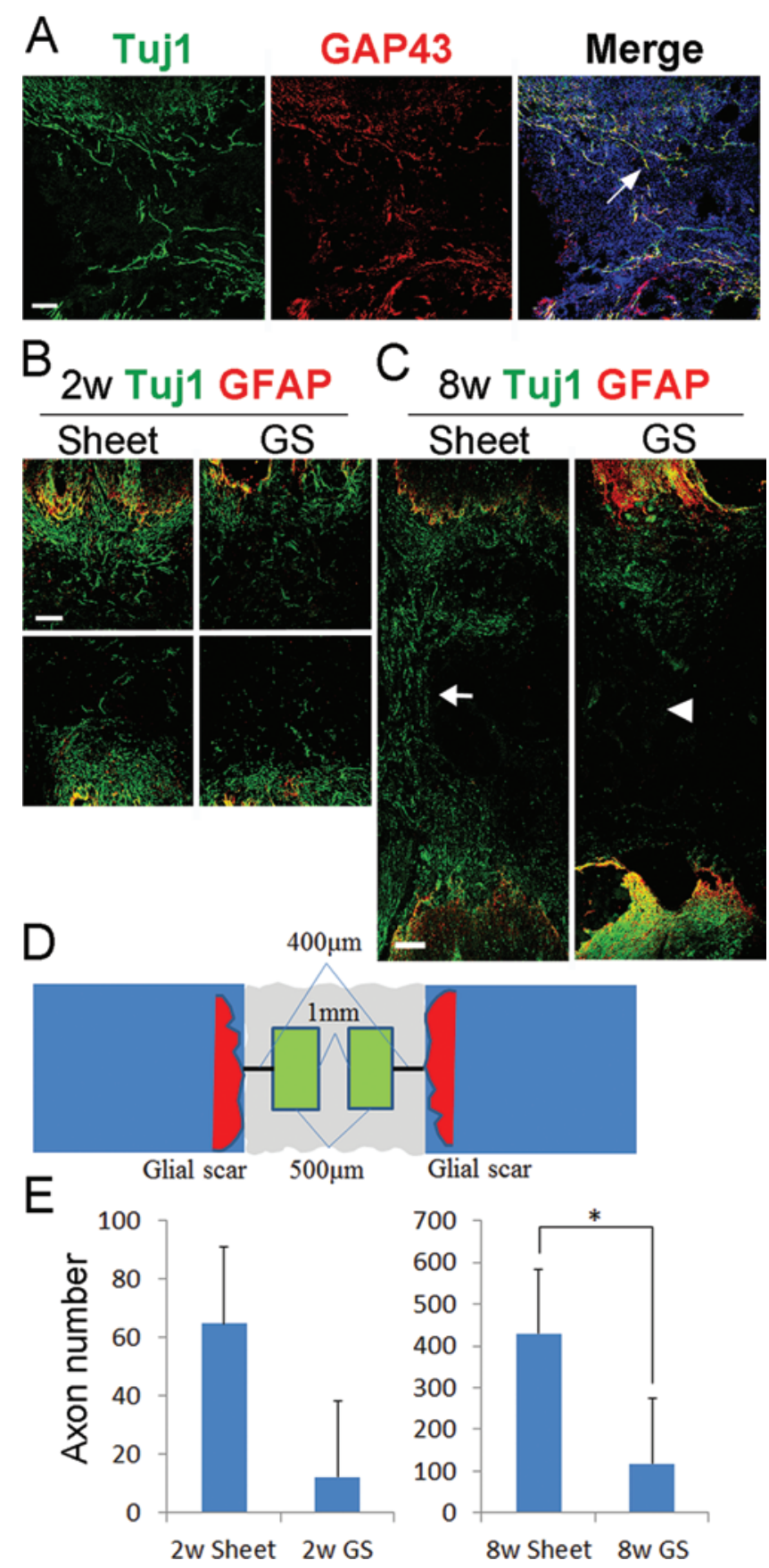

FIG. 3. The transplantation of BMSC sheets promoted axonal regeneration across the BMSC sheet-transplanted site. All sections were sliced in the coronal plane. A: The majority of Tuj1-positive axons at the sheet-transplanted site overlapped with GAP43-positive axons at 2 weeks after transplantation (arrow). B and C: Many more Tuj1positive axons beyond the GFAP-positive glial scar were observed at the sheet-transplanted site compared with the GS-transplanted site at 2 weeks (2w; B [upper: rostral; lower: caudal]) and 8 weeks (8w; C) after transplantation. At 8 weeks after transplantation, elongated Tuj1-positive axons across the transplanted site were observed only in the sheettransplanted animals (arrow), but not in the GS-transplanted animals (arrowhead). D: The number of axons with a length of at least $50 \mu \mathrm{m}$ was counted within the 2 rectangles $400 \mu \mathrm{m}$ away from the rostral or caudal stump of the injury site. The counting areas are within the green boxes. Fig. 3. (continued) $\rightarrow$ shape lengthwise in the rostrocaudal direction in sheettransplanted animals, whereas it showed a tightly packed shape with the spinal cords walled off from the transplant at the rostral and caudal stumps in GS-transplanted animals (Fig. 4C). GFAP-positive areas (black-outlined boxes in Fig. 4D) in the sheet group were significantly reduced compared with those of the GS group at both time points (Fig. 4E; at 2 weeks: BMSC, 799,429 $\pm 71,588 \mu \mathrm{m}^{2}$; GS, $159,0592 \pm 190,938 \mu \mathrm{m}^{2} ; \mathrm{p}=0.008, \mathrm{n}=4$; and at 8 weeks: BMSC, 403,005 $\pm 95,128 \mu \mathrm{m}^{2}$; GS: $130,5847 \pm 186,634$ $\mu \mathrm{m}^{2} ; \mathrm{p}=0.005, \mathrm{n}=4$ per group). These results indicated that the BMSC sheets attenuated glial scar formation after SCI and affected the shape of reactive astrocytes.

\section{BMSC Sheets Improved Locomotor Function Compared with GS}

The BBB scores were measured at $0,1,2,4$, and 8 weeks after transplantation (Fig. 5). Significant differences in BBB scores between the sheet and GS groups were observed at 4 weeks (sheet: $5.13 \pm 0.32$; GS: $2.75 \pm 0.48$; $\mathrm{p}<0.05, \mathrm{n}=6$ ) and 8 weeks (sheet: $5.25 \pm 0.14$; GS: 3.00 $\pm 0.29 ; \mathrm{p}<0.05$ vs GS, $\mathrm{n}=6$ ) after transplantation. These results indicated that the BMSC sheet improved locomotor function compared with GS transplantation.

\section{Discussion}

Several studies have demonstrated that transplantation of BMSCs (nonsheet form) is effective for axonal regeneration and enhanced hindlimb motor function in both contusive ${ }^{4,8,24}$ and transected ${ }^{33} \mathrm{SCI}$ rat models. The present study demonstrates that the sheet form of BMSCs is also effective for promoting axonal regeneration and functional improvement in a transected SCI model. Transplanted BMSCs do not tend to differentiate into neural cells in the host's central nervous system..$^{6,9}$ Thus, the observed regenerating axons from the rostral to caudal stumps are considered to be the hosts' neurons.

Recent evidence has shown that the inhibition of axonal regeneration is mainly due to the formation of a glial scar, which is a type of secondary damage following SCI. ${ }^{34}$ The present study also showed that BMSC sheets suppress glial scar formation in the same manner as observed for the nonsheet form of BMSCs. ${ }^{1,10,24,30}$ Considered together with the result of the coculture experiment of the sheet and neurospheres, BMSC sheets seem to provide a permissive environment for injured axons. Recent evidence suggests that a glial scar formed by GFAP-positive astrocytes does not always inhibit axonal regeneration, but rather the shape of the scar is a more important factor for its inhibitory effect. ${ }^{29}$ The same study described that the penetration of astrocyte processes into a transplant is an indicator of a permissive environment for axonal regeneration. Our results showed that GFAP-positive processes were elongating toward the sheet-transplanted site, especially at 8 weeks after transplantation. In contrast, these features were not observed in the GS-transplanted site,

FIG. 3. E: The numbers of axons obtained from 9 sections at $80-\mu \mathrm{m}$ intervals were summed. There were 4 rats in each group. ${ }^{*} p<0.05$, Welch's test. Bar $=200 \mu \mathrm{m}$. Figure is available in color online only. 


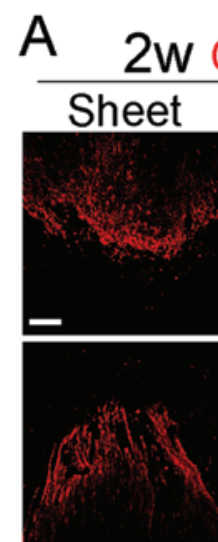

$\bar{C}$
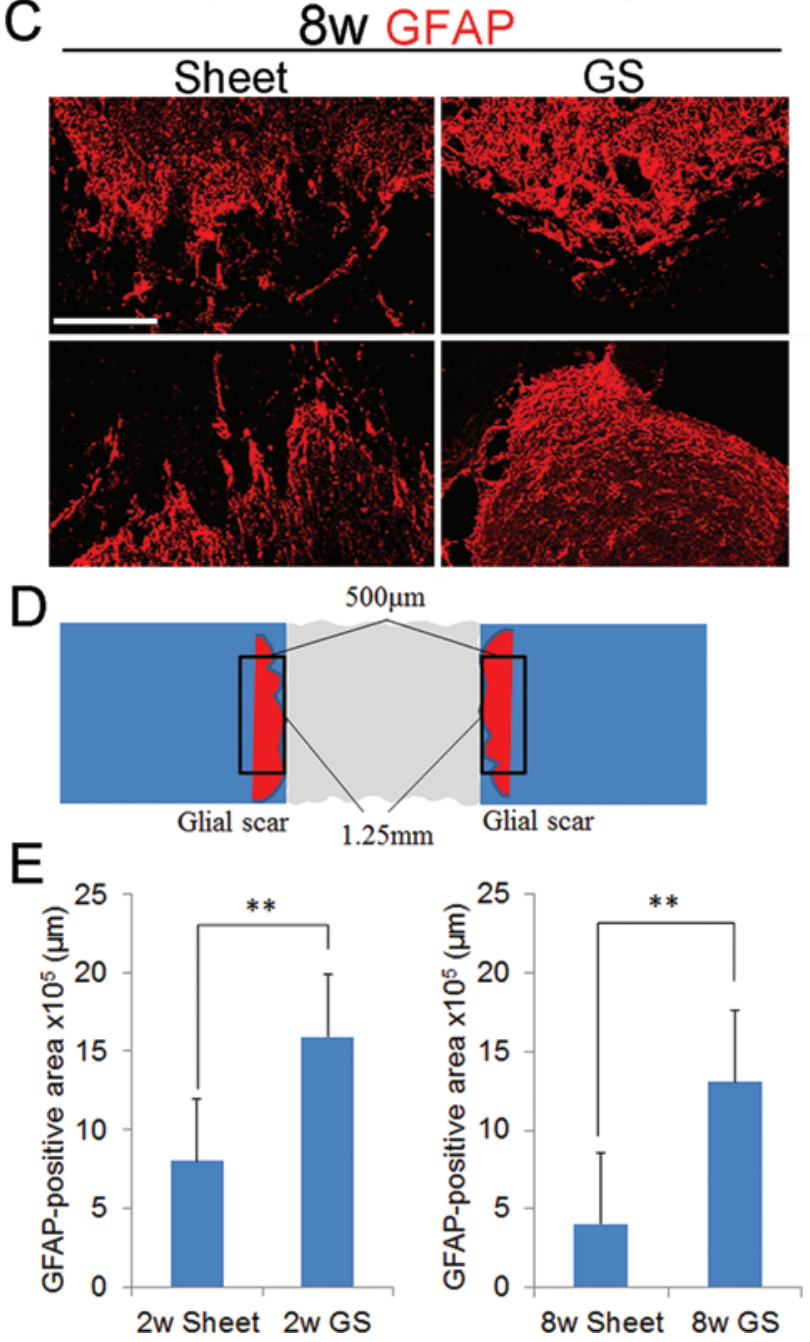

FIG. 4. BMSC sheets inhibited glial scar formation and resulted in an irregular glial scar shape. A and B: GFAP immunoreactivity in the rostral and caudal stumps of the spinal cord appeared to be weaker in the sheet-transplanted animals than in the GS-transplanted animals at 2 and 8 weeks after transplantation (upper: rostral sides; lower: caudal sides). C: At 8 weeks after transplantation, high-magnification images of GFAP immunoreactivity showed irregular shapes lengthwise in the rostrocaudal direction in the sheet-transplanted animals, and sharp shapes at the rostral and caudal stumps in the GS-transplanted animals. D: GFAP-positive areas within the 2 rectangles including the rostral or caudal edge of the injury site are shown. The calculated area is in the black-outlined boxes. Fig. 4. (continued) $\rightarrow$

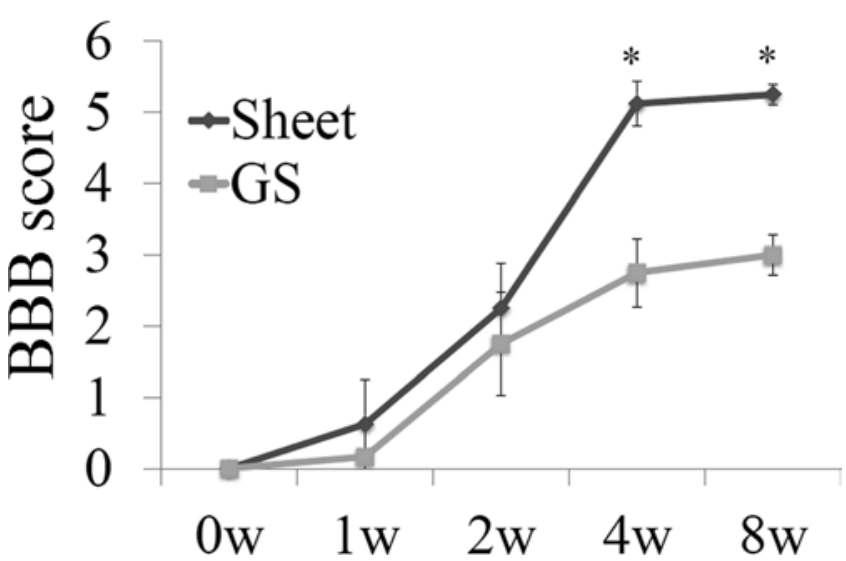

FIG. 5. The hindlimb locomotor assessment. The graph shows BBB scores in sheet-transplanted and GS-transplanted animals 6 rats from each group) at 1, 2, 4, and 8 weeks after transplantation. Data are presented as the mean \pm SEM. ${ }^{*} p<0.05$ vs GS.

where tightly packed GFAP-positive astrocytes walled off the spinal cord from the transplant. These results demonstrate that BMSC sheets not only suppress glial scar formation but also likely provide a good environment for the regeneration of injured axons by affecting the morphology of reactive astrocytes.

Cell sheet technology has attracted broad attention in tissue engineering because transplanted cell sheets can maintain their biological activity and prevent the spilling out of transplanted cells without scaffolds. Most cases of SCI in clinical settings result from contusion, and the chronic phase involves the formation of cavities and a glial scar. Although the present study used a transection model of SCI, we suppose that BMSC sheets would also be clinically useful for filling these cavities. Another possible usage of BMSC sheets is to fill the defect formed after resection of the glial scar, because the scar can be the wall inhibiting regenerating axons even after cell transplantation. Finally, the present study showed that coculture of neurospheres and BMSC sheets is possible, which highlights the potential for the coculture of BMSC sheets and neural stem cells derived from induced pluripotent stem cells as a great step toward clinical application. Further studies are needed before clinical trials of BMSC sheets can be conducted, such as experiments using nonhuman primates.

\section{Conclusions}

The present study indicates the potential therapeutic effects of BMSC sheets for SCI, which would enable autologous transplantation without requiring scaffolds.

\section{Acknowledgments}

We thank Haruo Kanno, Satoshi Tateda, and Kenichiro Yahata (Tohoku University School of Medicine, Japan) for technical advice and Fumika Kunda and Yumi Moriwake (Nara Medical University

FIG. 4. E: GFAP-positive areas obtained from 9 sections at $80-\mu \mathrm{m}$ intervals were summed. There were 4 rats in each group. ${ }^{* *} p<0.01$ vs GS, t-test. Bar $=200 \mu \mathrm{m}$. Figure is available in color online only. 
School of Medicine, Japan) for technical assistance. This work was supported by JSPS KAKENHI Grant No. 15K10417 to Akinori Okuda, Noriko Horii-Hayashi, Takamasa Shimizu, Manabu Akahane, Mayumi Nishi, and Yasuhito Tanaka.

\section{References}

1. Abrams MB, Dominguez C, Pernold K, Reger R, WiesenfeldHallin Z, Olson L, et al: Multipotent mesenchymal stromal cells attenuate chronic inflammation and injury-induced sensitivity to mechanical stimuli in experimental spinal cord injury. Restor Neurol Neurosci 27:307-321, 2009

2. Akahane M, Nakamura A, Ohgushi H, Shigematsu H, Dohi Y, Takakura Y: Osteogenic matrix sheet-cell transplantation using osteoblastic cell sheet resulted in bone formation without scaffold at an ectopic site. J Tissue Eng Regen Med 2:196-201, 2008

3. Akahane M, Shigematsu H, Tadokoro M, Ueha T, Matsumoto T, Tohma Y, et al: Scaffold-free cell sheet injection results in bone formation. J Tissue Eng Regen Med 4:404-411, 2010

4. Ankeny DP, McTigue DM, Jakeman LB: Bone marrow transplants provide tissue protection and directional guidance for axons after contusive spinal cord injury in rats. Exp Neurol 190:17-31, 2004

5. Basso DM, Beattie MS, Bresnahan JC, Anderson DK, Faden AI, Gruner JA, et al: MASCIS evaluation of open field locomotor scores: effects of experience and teamwork on reliability. Multicenter Animal Spinal Cord Injury Study. J Neurotrauma 13:343-359, 1996

6. Castro RF, Jackson KA, Goodell MA, Robertson CS, Liu H, Shine HD: Failure of bone marrow cells to transdifferentiate into neural cells in vivo. Science 297:1299, 2002

7. Chen MH, Chen PR, Chen MH, Hsieh ST, Huang JS, Lin FH: An in vivo study of tricalcium phosphate and glutaraldehyde crosslinking gelatin conduits in peripheral nerve repair. $\mathbf{J}$ Biomed Mater Res B Appl Biomater 77:89-97, 2006

8. Chopp M, Zhang XH, Li Y, Wang L, Chen J, Lu D, et al: Spinal cord injury in rat: treatment with bone marrow stromal cell transplantation. Neuroreport 11:3001-3005, 2000

9. Coyne TM, Marcus AJ, Woodbury D, Black IB: Marrow stromal cells transplanted to the adult brain are rejected by an inflammatory response and transfer donor labels to host neurons and glia. Stem Cells 24:2483-2492, 2006

10. Dasari VR, Veeravalli KK, Dinh DH: Mesenchymal stem cells in the treatment of spinal cord injuries: A review. World J Stem Cells 6:120-133, 2014

11. Davis JB, Stroobant P: Platelet-derived growth factors and fibroblast growth factors are mitogens for rat Schwann cells. J Cell Biol 110:1353-1360, 1990

12. Hokari M, Kuroda S, Shichinohe H, Yano S, Hida K, Iwasaki Y: Bone marrow stromal cells protect and repair damaged neurons through multiple mechanisms. J Neurosci Res 86:1024-1035, 2008

13. Horii-Hayashi N, Sasagawa T, Hashimoto T, Kaneko T, Takeuchi K, Nishi M: A newly identified mouse hypothalamic area having bidirectional neural connections with the lateral septum: the perifornical area of the anterior hypothalamus rich in chondroitin sulfate proteoglycans. Eur J Neurosci 42:2322-2334, 2015

14. Horii-Hayashi N, Sasagawa T, Matsunaga W, Nishi M: Development and structural variety of the chondroitin sulfate proteoglycans-contained extracellular matrix in the mouse brain. Neural Plast 2015:256389, 2015

15. Huang YC, Huang YY: Biomaterials and strategies for nerve regeneration. Artif Organs 30:514-522, 2006

16. Ide C, Nakai Y, Nakano N, Seo TB, Yamada Y, Endo K, et al: Bone marrow stromal cell transplantation for treatment of sub-acute spinal cord injury in the rat. Brain Res 1332:3247, 2010

17. Ju YE, Janmey PA, McCormick ME, Sawyer ES, Flanagan
LA: Enhanced neurite growth from mammalian neurons in three-dimensional salmon fibrin gels. Biomaterials 28:2097-2108, 2007

18. Kamada T, Koda M, Dezawa M, Anahara R, Toyama Y, Yoshinaga K, et al: Transplantation of human bone marrow stromal cell-derived Schwann cells reduces cystic cavity and promotes functional recovery after contusion injury of adult rat spinal cord. Neuropathology 31:48-58, 2011

19. Kang KN, Lee JY, Kim DY, Lee BN, Ahn HH, Lee B, et al: Regeneration of completely transected spinal cord using scaffold of poly(D,L-lactide-co-glycolide)/small intestinal submucosa seeded with rat bone marrow stem cells. Tissue Eng Part A 17:2143-2152, 2011

20. Kira T, Omokawa S, Akahane M, Shimizu T, Nakano K, Nakanishi Y, et al: Effectiveness of bone marrow stromal cell sheets in maintaining random-pattern skin flaps in an experimental animal model. Plast Reconstr Surg 136:624e-632e, 2015

21. Lalu MM, McIntyre L, Pugliese C, Fergusson D, Winston BW, Marshall JC, et al: Safety of cell therapy with mesenchymal stromal cells (SafeCell): a systematic review and metaanalysis of clinical trials. PLoS One 7:e47559, 2012

22. Mannari T, Sawa H, Furube E, Fukushima S, Nishikawa K, Nakashimna T, et al: Antidepressant-induced vascular dynamics in the hippocampus of adult mouse brain. Cell Tissue Res 358:43-55, 2014

23. Nakamura A, Akahane M, Shigematsu H, Tadokoro M, Morita Y, Ohgushi H, et al: Cell sheet transplantation of cultured mesenchymal stem cells enhances bone formation in a rat nonunion model. Bone 46:418-424, 2010

24. Neuhuber B, Timothy Himes B, Shumsky JS, Gallo G, Fischer I: Axon growth and recovery of function supported by human bone marrow stromal cells in the injured spinal cord exhibit donor variations. Brain Res 1035:73-85, 2005

25. Ren J, Jin P, Sabatino M, Balakumaran A, Feng J, Kuznetsov SA, et al: Global transcriptome analysis of human bone marrow stromal cells (BMSC) reveals proliferative, mobile and interactive cells that produce abundant extracellular matrix proteins, some of which may affect BMSC potency. Cytotherapy 13:661-674, 2011

26. Spilker MH, Yannas IV, Kostyk SK, Norregaard TV, Hsu HP, Spector M: The effects of tubulation on healing and scar formation after transection of the adult rat spinal cord. Restor Neurol Neurosci 18:23-38, 2001

27. Toritsuka M, Kimoto S, Muraki K, Landek-Salgado MA, Yoshida A, Yamamoto N, et al: Deficits in microRNA-mediated Cxcr4/Cxcl12 signaling in neurodevelopmental deficits in a 22q11 deletion syndrome mouse model. Proc Natl Acad Sci U S A 110:17552-17557, 2013

28. Urdzíková L, Jendelová P, Glogarová K, Burian M, Hájek M, Syková E: Transplantation of bone marrow stem cells as well as mobilization by granulocyte-colony stimulating factor promotes recovery after spinal cord injury in rats. J Neurotrauma 23:1379-1391, 2006

29. Williams RR, Henao M, Pearse DD, Bunge MB: Permissive Schwann cell graft/spinal cord interfaces for axon regeneration. Cell Transplant 24:115-131, 2015

30. Wright KT, El Masri W, Osman A, Chowdhury J, Johnson WE: Concise review: Bone marrow for the treatment of spinal cord injury: mechanisms and clinical applications. Stem Cells 29:169-178, 2011

31. Yamaya S, Ozawa H, Kanno H, Kishimoto KN, Sekiguchi A, Tateda S, et al: Low-energy extracorporeal shock wave therapy promotes vascular endothelial growth factor expression and improves locomotor recovery after spinal cord injury. $\mathbf{J}$ Neurosurg 121:1514-1525, 2014

32. Yang J, Yamato M, Nishida K, Ohki T, Kanzaki M, Sekine $\mathrm{H}$, et al: Cell delivery in regenerative medicine: the cell sheet engineering approach. J Control Release 116:193-203, 2006 
33. Zeng X, Zeng YS, Ma YH, Lu LY, Du BL, Zhang W, et al: Bone marrow mesenchymal stem cells in a three-dimensional gelatin sponge scaffold attenuate inflammation, promote angiogenesis, and reduce cavity formation in experimental spinal cord injury. Cell Transplant 20:1881-1899, 2011

34. Zhang LX, Yin YM, Zhang ZQ, Deng LX: Grafted bone marrow stromal cells: a contributor to glial repair after spinal cord injury. Neuroscientist 21:277-289, 2015

\section{Disclosures}

The authors report no conflict of interest concerning the materials or methods used in this study or the findings specified in this paper.

\section{Author Contributions}

Conception and design: Okuda, Horii-Hayashi, Shimizu, Koizumi, Akahane, Nishi, Tanaka. Acquisition of data: Okuda, Sasagawa, Morimoto, Masuda. Analysis and interpretation of data: Okuda, Horii-Hayashi, Nishi. Drafting the article: Okuda, Horii-Hayashi. Critically revising the article: Okuda, Horii-Hayashi. Reviewed submitted version of manuscript: Okuda, HoriiHayashi. Approved the final version of the manuscript on behalf of all authors: Okuda. Statistical analysis: Okuda, Akahane.

Administrative/technical/material support: Sasagawa, Shimizu, Shigematsu, Iwata, Morimoto, Masuda. Study supervision: Okuda, Shimizu, Shigematsu, Iwata, Koizumi, Akahane, Nishi, Tanaka.

\section{Correspondence}

Akinori Okuda, Department of Orthopaedic Surgery, Nara Medical University, Kashihara, Nara 634-8522, Japan. email: okuda74@naramed-u.ac.jp. 